\title{
Review
}

\section{The role of ALFY in selective autophagy}

\author{
P Isakson ${ }^{1}$, P Holland ${ }^{1}$ and A Simonsen ${ }^{\star, 1}$
}

Autophagy, a highly conserved lysosomal degradation pathway, was initially characterized as a bulk degradation system induced in response to starvation. In recent years, autophagy has emerged also as a highly selective pathway, targeting various cargoes such as aggregated proteins and damaged organelles for degradation. The key factors involved in selective autophagy are autophagy receptors and adaptor proteins, which connect the cargo to the core autophagy machinery. In this review, we discuss the current knowledge about the only mammalian adaptor protein identified thus far, autophagy-linked FYVE protein (ALFY). ALFY is a large, scaffolding, multidomain protein implicated in the selective degradation of ubiquitinated protein aggregates by autophagy. We also comment on the possible role of ALFY in the context of disease.

Cell Death and Differentiation (2013) 20, 12-20; doi:10.1038/cdd.2012.66; published online 1 June 2012

\section{Facts}

- Selective autophagy relies upon specific autophagy receptors and adaptor proteins that link the cargo with the core autophagic machinery.

- The autophagy receptor p62 interacts directly with ubiquitin and with microtubule-associated protein 1 light chain 3, thereby facilitating autophagic degradation of ubiquitinated cargo.

- p62 interacts directly with the adaptor protein ALFY and both are involved in the formation and autophagic degradation of ubiquitinated protein aggregates.

- ALFY links ubiquitinated proteins to the core autophagic machinery through interactions of its C-terminal Pleckstrin homology-Beige and Chediak-Higashi domain with p62, its WD40 domain with autophagy-related gene (Atg5) and its FYVE domain with phosphatidylinositol 3-phosphate.

- Overexpression of an ALFY C-terminal fragment led to a significant reduction of protein aggregates in a primary neuronal model of Huntington's disease and was neuroprotective in a Drosophila eye model of polyQ disease.

\section{Open Questions}

- How are the various types of selective autophagy regulated?

- What are the ligands targeting different cargoes for autophagic degradation?

- Which autophagy receptors and adaptor proteins are involved in recognition of the various types of cargo for selective autophagy?
- Is ALFY required for other types of selective autophagy than aggrephagy?

- What is the role of ALFY and p62 nucleocytoplasmic shuttling?

Autophagy is an evolutionary conserved catabolic pathway found in all eukaryotes involving delivery of cytoplasmic components to the lysosome for degradation. Macroautophagy (hereafter referred to as autophagy) involves nucleation of a unique membrane structure (the phagophore), which expands around parts of the cytoplasm to form a double-membrane vesicle called the autophagosome. Autophagosomes can fuse directly with the lysosome or first with endocytic vesicles, generating amphisomes, which eventually fuse with lysosomes where the sequestered material is degraded by lysosomal hydrolases. The degradation products are recycled back to the cytoplasm to be used as building blocks for new macromolecules and for energy production. The identification of about 30 conserved autophagy-related gene $(A t g)$ products in the previous decade has provided a growing understanding of the complex process of autophagy. The Atg proteins predominantly make up the core autophagic machinery required for phagophore nucleation and expansion, including four major multi-protein complexes that have been reviewed in detail elsewhere. ${ }^{1}$ Briefly, the ULK1 complex, including Atg13, FIP200 and Atg101, is required for phagophore nucleation. ${ }^{2}$ The nucleation additionally requires the class III phosphatidylinositol 3-kinase (PI3K) complex I, containing the catalytic and regulatory subunits Vps34 and Vps15, as well as Beclin 1 and ATG14L. ${ }^{3}$ The integral membrane protein $\mathrm{mAtg} 9$ must also be recruited

\footnotetext{
${ }^{1}$ Department of Biochemistry, Institute of Basic Medical Sciences, University of Oslo, Oslo, Norway

*Corresponding author: A Simonsen, Department of Biochemistry, Institute of Basic Medical Sciences, University of Oslo, PO Box 1112 Blindern, 0317, Oslo, Norway. Tel: + 47 22851110; Fax: + 47 22851058; E-mail:anne.simonsen@medisin.uio.no

Keywords: selective autophagy; ALFY; p62; protein aggregates; BEACH domain

Abbreviations: ALFY, autophagy-linked FYVE protein; Ape1, aminopeptidase 1; APL, acute promyelocytic leukemia; Atg, autophagy-related gene; ATO, arsenic trioxide; ATRA, all-trans retinoic acid; BEACH, Beige and Chediak-Higashi; Bchs, blue cheese; Cvt, cytoplasm to vacuole targeting; FAN, factor associated with neural sphingomyelinase; GAP, GTPase-activating proteins; GOF, gain-of-function; Htt, huntingtin; HD, Huntington's disease; LC3, MAP1 light chain 3; LIR, LC3-interacting region; NES, nuclear export signal; NLS, nuclear localization signal; PAS, pre-autophagosomal structure; PB1, Phox and Bem1p; PE, phosphatidylethanolamine; $\mathrm{PH}$, Pleckstrin homology; PI3K, phosphatidylinositol 3-kinase; PI3P, phosphatidylinositol 3-phosphate; PML-NBs, promyelocytic leukemia nuclear bodies; UPS, ubiquitin-proteasome system

Received 01.2.12; revised 12.4.12; accepted 18.4.12; Edited by M Piacentini; published online 01.6.12
} 
to the site of phagophore nucleation, but its exact function is still unknown. ${ }^{4}$ Finally, the two ubiquitin-like proteins Atg12 and microtubule-associated protein 1 (MAP1) (MAP1 light chain 3 (LC3)) (the homolog of yeast Atg8) and their conjugation systems are required for autophagosome formation. ${ }^{5,6}$ Analogous to ubiquitination, Atg12 and LC3 are activated by an E1-like enzyme (Atg7) and conjugated by an E2-like enzyme (Atg10 and Atg3, respectively) to Atg5 or phosphatidylethanolamine (PE), respectively. ${ }^{7}$ The Atg12-Atg5 conjugate associates with the membrane-bound protein Atg16 $\mathrm{L}$ to form a high-molecular-weight complex. The Atg12-Atg5-Atg16L complex seems to determine the site of LC3 lipidation and work in an E3-like manner for conjugation of LC3 to PE. ${ }^{8,9}$

\section{Selective Autophagy}

It is becoming increasingly evident that autophagy is also a highly selective process and that basal levels of autophagy serves a quality control function by selective elimination of specific cellular structures, which could cause cellular damage if not properly removed. Selective autophagy was first described in yeast. The cytoplasm to vacoule targeting (Cvt) pathway involves selective transport of some vacuolar hydrolases from the cytosol to the yeast vacuole ${ }^{10}$ and can serve as a model for selective autophagy in higher organisms. A number of organelles have been found to be selectively turned over by autophagy and cargo-specific names have been given to distinguish the various types of selective pathways (Figure 1). ${ }^{11}$ Some examples include degradation of the ER (reticulophagy or ERphagy), peroxisomes (pexophagy), mitochondria (mitophagy), lipid droplets (lipophagy), zymogen granules (zymophagy) and even parts of the nucleus (nucleophagy). Moreover, pathogens (xenophagy), ribosomes (ribophagy), glycogen particles (glycophagy) and aggregation-prone proteins (aggrephagy) are targeted for degradation by selective autophagy. To perform this quality control function, selective types of autophagy must be able to distinguish cargoes ready for degradation from their functional counterparts. The molecular machinery involved in the regulation of the different forms of selective autophagy is just beginning to become revealed, but it is evident that specific cargo is targeted for degradation by the action of specific cargo-recognizing receptors (called autophagy receptors) and specificity adaptors, which together function to bring the cargo in contact with the core autophagic machinery to allow formation of the autophagosomal membrane around the cargo (Figure 1). Although selective autophagy relies on the same molecular core machinery as starvation-induced autophagy, autophagy receptors and adaptors in general seem not to be required for non-selective autophagy.

\section{Autophagy Receptors and Cargo Ligands}

Autophagy receptors are defined as proteins being able to interact directly with both the cargo to become degraded and an Atg8/LC3 family member through a specific (WxxL) motif, ${ }^{12}$ commonly referred to as the LC3-interacting region (LIR) ${ }^{13}$ or the LC3 recognition sequence motif (Figure 1). ${ }^{14}$ The Atg8/ LC3 family includes LC3A, LC3B, LC3B2, LC3C, GABARAP,

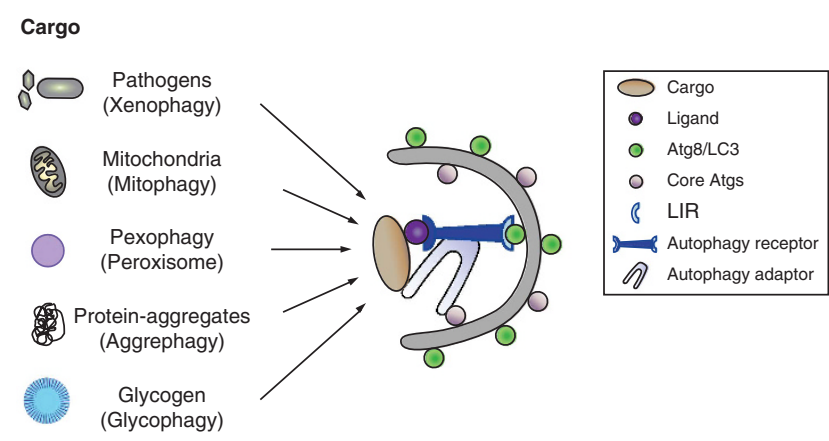

Figure 1 Model for selective autophagy. Selective autophagy depends on autophagy receptors and adaptors linking the cargo to become degraded to the autophagic membranes. The cargo is recognized by an autophagy receptor that has a specific ligand-binding domain (e.g. an ubiquitin-binding domain) as well as a motif known as LIR, mediating its interaction with Atg8/LC3 family proteins. An adaptor protein (e.g. ALFY) further facilitate sequestration of the specific cargo by linking the cargo-receptor complex to core Atg proteins. Cargo-specific names have been given to describe the various types of selective autophagy, including xenophagy (invasive pathogens), mitophagy (mitochondria), pexophagy (peroxisomes), aggrephagy (aberrant protein aggregates and disease-related inclusions) and glycophagy (glycogen particles)

GABARAP-L1/GEC-1 and GATE-16/GABARAP-L2/GEF2 ${ }^{15}$ and whereas most autophagy receptors have been found to interact with several family members in vitro their in vivobinding specificities remain elusive. It should also be pointed out that not all LIR-containing proteins are autophagy receptors. Some LIR-containing proteins are recruited to autophagic membranes to facilitate autophagosome formation, ${ }^{16,17}$ whereas others interact with LC3 to facilitate autophagosome transport and maturation ${ }^{18,19}$ or use their LIR motif to be targeted for degradation themselves. ${ }^{20}$

The list of autophagy receptors is rapidly expanding and it is also becoming evident that a particular cargo may be recognized by several autophagy receptors. Autophagy receptors have so far been implicated in the degradation of bacteria (p62/SQSTM1, ${ }^{21-23}$ NDP $52^{24}$ and optineurin ${ }^{25}$ ), viral particles (p62), ${ }^{26}$ mitochondria (p62, Nix/Bnip3L and Atg32), ${ }^{27-31}$ peroxisomes (p62 and Atg30), ${ }^{32,33}$ midbody remnant (p62 and NBR1), ${ }^{34,35}$ glycogen particles ${ }^{36}$ and in the Cvt pathway (Atg19 and Atg34). ${ }^{10,37,38}$

The ligands responsible for selective recognition of cargo can be defined as a molecular entity on the surface of a cargo recognized by an autophagy receptor (Figure 1). Such ligands are yet largely unknown, but have been identified for some autophagy cargoes. Addition of ubiquitin to cargo-specific proteins on mitochondria, peroxisomes, ${ }^{39}$ protein aggregates $^{40}$ and bacteria ${ }^{41}$ can serve as a signal for selective recognition of such structures by autophagy receptors. The autophagy receptors p62, NBR1, NDP52 and optineurin all have ubiquitin-interacting domains, as well as a LIR motif, and thereby facilitate autophagic degradation of ubiquitinated cargo. ${ }^{40,41}$ However, neither the identity of the cargo proteins becoming ubiquitinated, nor the ligases or the type of ubiquitin modification involved are known in most cases. Moreover, it is clear that some cargo (e.g. mitochondria) can be targeted for selective autophagy independently of ubiquitin, and also that autophagy receptors as p62 can directly recognize cargo independently of ubiquitin. ${ }^{40}$ p62 is the first described and 
best studied autophagy receptor and its role in targeting protein aggregates for autophagic degradation will be discussed in more detail below. ${ }^{42}$

\section{Adaptor Proteins}

The adaptor proteins are less well described, but can be defined as scaffolding proteins, which interact both with the cargo-autophagy receptor complex and with components of the core Atg machinery to facilitate formation of the autophagosomal membrane around the cargo to be degraded (Figure 1). The best described adaptor protein is yeast Atg11, involved in the Cvt pathway, ${ }^{43}$ mitophagy ${ }^{28,44}$ and pexophagy. ${ }^{45}$

The Cvt pathway is a biosynthetic process involving transport of some vacuolar hydrolases (e.g. the precursor aminopeptidase 1 (prApe1) complex) from the cytosol to the vacuole. The cytosolic Apel complex is recognized by the autophagy receptor Atg19. Before binding to Atg8, the prApe1-Atg19 complex binds directly to Atg11, which transports the complex to the pre-autophagosomal structure (PAS) through an interaction with the actin cytoskeleton. ${ }^{43}$ Atg11 also interacts with Atg9 and the Atg1/ULK complex and thereby serves to scaffold the machinery required for sequestration of the Apel complex into small, autophagosome-like, Cvt vesicles. ${ }^{46}$ Interestingly, increased Atg11 expression facilitates recruitment of Atg8 and Atg9 to the
PAS and formation of more Cvt vesicles, suggesting that Atg11 can regulate the rate of selective autophagy. ${ }^{10}$ Atg11 interacts also with the autophagy receptors Atg30 (pexophagy) $^{45}$ and Atg32 (mitophagy) ${ }^{28,44}$ and likely has a similar role in selective autophagy of these cargo, but it is not required for non-selective starvation-induced autophagy.

Similarly to the lack of homology between the mammalian autophagy receptors and Atg19, mammalian cells appear to not possess an Atg11 homolouge. Instead, the large scaffolding protein autophagy-linked FYVE protein (ALFY) has a similar function in selective autophagy in mammalian cells. ALFY interacts directly with the autophagy receptor p62 and with the core autophagy protein Atg5, as well as with phosphatidylinositol 3-phosphate (PI3P) found in autophagic membranes, suggesting that it functions as a scaffolding protein. $^{47-49}$

\section{ALFY Domain Structure}

ALFY (also called WDFY3; WD repeat and FYVE domain-containing protein 3 ) is a 3526 residue protein with a predicted $\mathrm{Mr}$ of $395 \mathrm{kDa}$. The gene encoding ALFY is located on chromosome 4q21 (NCBI Human Gene Map) and is composed of 68 exons. ${ }^{47}$ Alternative transcript variants are predicted to exist, but it is not known whether they encode functional proteins. The N-terminal two thirds of ALFY contains no predicted functional domains, but there are
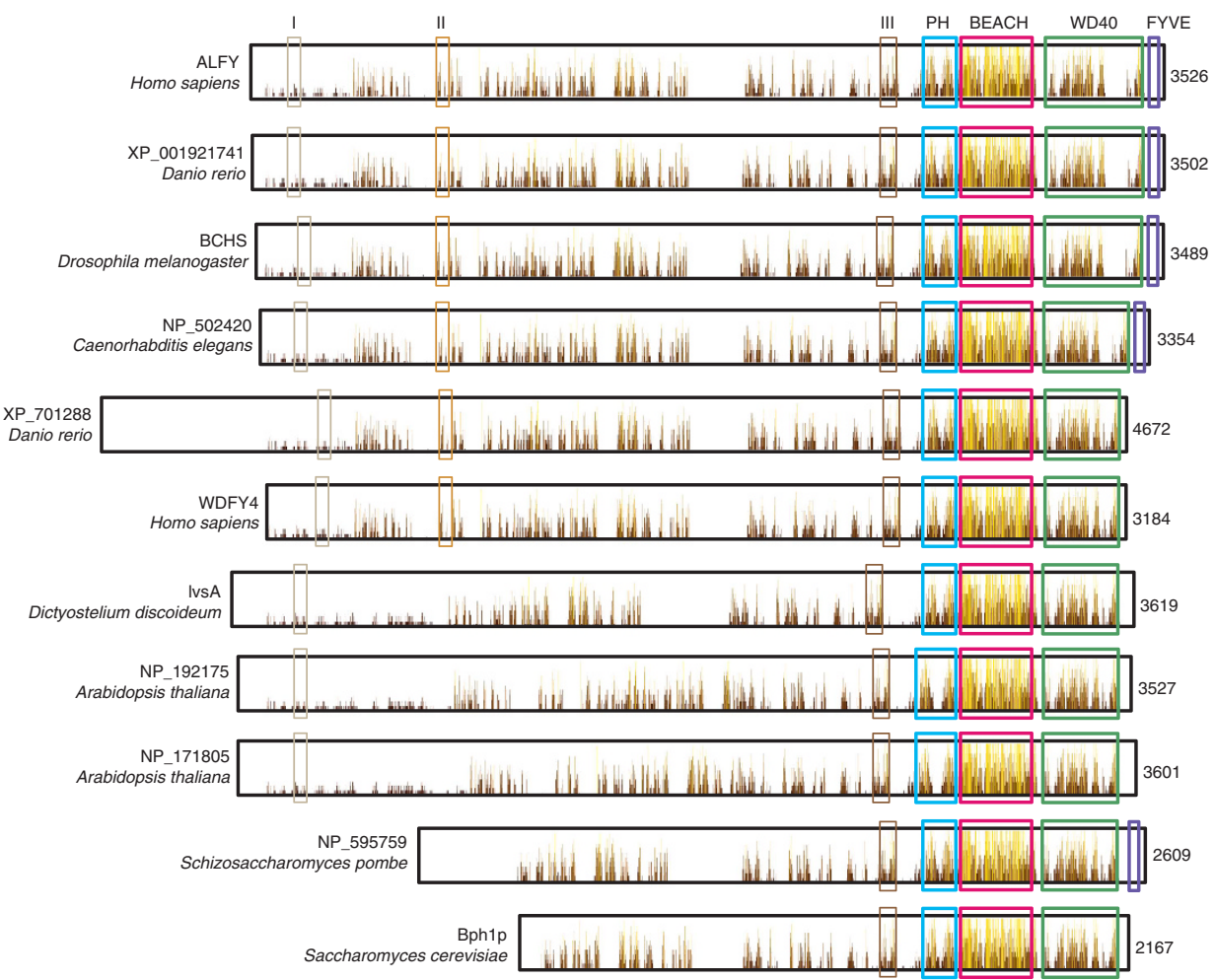

Figure 2 Domain structure and degree of sequence conservation in proteins most closely related to ALFY. Inside each protein box, bars illustrate the degree of sequence conservation throughout the indicated proteins, higher and more yellow bars indicate higher degree of conservation. Gray, light brown and dark brown boxes indicate regions of significant sequence homology to unrelated proteins, residues 247-351 (of ALFY) in gray [I] is similar to 'TBC1 domain family member 30', residues 842-923 in light brown [II] is similar to 'origin recognition complex subunit 2' and residues 2281-2397 in dark brown [III] is similar to 'BCL-2-associated transcription factor 1'. Blue boxes indicate $\mathrm{PH}$ domains, red BEACH domains, green WD40 domains and purple FYVE domains 
regions showing considerable homology to functional domains in other proteins (Figure 2, indicated as regions of interest I, II and III). Most notable of these is region I (residues 247-351 of ALFY) that is similar to a large part of the TBC domain of 'TBC1 domain family member 30'. The TBC domain is found in a wide range of different proteins including GTPase-activating proteins (GAPs), ${ }^{50}$ suggesting that ALFY might have GAP activity. Other regions of interest include residues 842-923 [II], similar to part of the 'origin recognition complex subunit 2' and 2281-2397 [III] that is similar to a part of the 'BCL-2-associated transcription factor 1'. The mentioned regions are generally conserved in the proteins most closely related to ALFY (Figure 2). The C-terminal region of ALFY contains several domains implicated in membrane trafficking; a typical FYVE domain, five WD40 repeats and a Pleckstrin homology (PH)-Beige and Chediak-Higashi $(\mathrm{BEACH})$ domain assembly (Figure 2). The FYVE domain is a zinc finger shown to interact specifically with PI3P. ${ }^{51}$ Most members of the FYVE family localize to early endosomes and multi-vesicular bodies, structures highly enriched in PI3P. ${ }^{52}$ The FYVE domain of ALFY interacts specifically with PI3P, but shows little or no colocalization with endosomes. ALFY rather colocalizes with ubiquitin- and p62-positive protein aggregates and to a certain degree with autophagic membranes. ${ }^{47}$ Recruitment of ALFY to protein aggregates seems not to depend on its binding to PI3P. ${ }^{47}$ However, as the class III PI3K/Vps34 complex, and by extension PI3P, is required for aggrephagy, ALFY may be involved in recruting PI3Pcontaining autophagic membranes to protein aggregates.
From sequence analysis ALFY is predicted to have five WD40 repeats, which are found to be essential for its interaction with Atg5. ${ }^{48}$ WD40 domains exist in a wide variety of eukaryotic proteins and typically form a seven bladed $\beta$ propeller structure to which proteins can bind either stably or reversibly. ${ }^{53}$ Most likely, the WD40 domain of ALFY can form a complete $\beta$-propeller structure without having seven WD40 repeats, but it also possible that WD40 repeats need to be donated from other interaction partners to complete the $\beta$-propeller. Finally, ALFY contains a $\mathrm{PH}$-like domain followed by a $\mathrm{BEACH}$ domain. ${ }^{54} \mathrm{PH}$ domains are generally involved in interaction with phosphoinositides or mediate protein-protein interactions. The $\mathrm{PH}$-like domain of the small human $\mathrm{BEACH}$ protein factor associated with neural sphingomyelinase (FAN) is shown to bind $\mathrm{PI}(4,5) \mathrm{P} 2,{ }^{55}$ but it is not known whether the $\mathrm{PH}$-like domain of ALFY is functional. The PH-like and WD40 domains show a moderate degree of conservation throughout human $\mathrm{BEACH}$ proteins whereas the $\mathrm{BEACH}$ domain is highly conserved. The BEACH domain is present in a family of proteins conserved throughout eukaryotes (Figure 3), but nothing is known about its function. In the case of FAN it has been shown that both the $\mathrm{PH}$-like and $\mathrm{BEACH}$ domains and the conserved interface between them are essential for its function, suggesting they function as a unit. ${ }^{54,56}$ Interestingly, we found the PH-BEACH domains of ALFY to interact with the autophagy receptor $\mathrm{p} 62 .{ }^{49}$

Hence, the C-terminal one third of ALFY contains several domains that together have the ability to connect cargo to the autophagic machinery; a p62-binding $\mathrm{PH}-\mathrm{BEACH}$ domain,

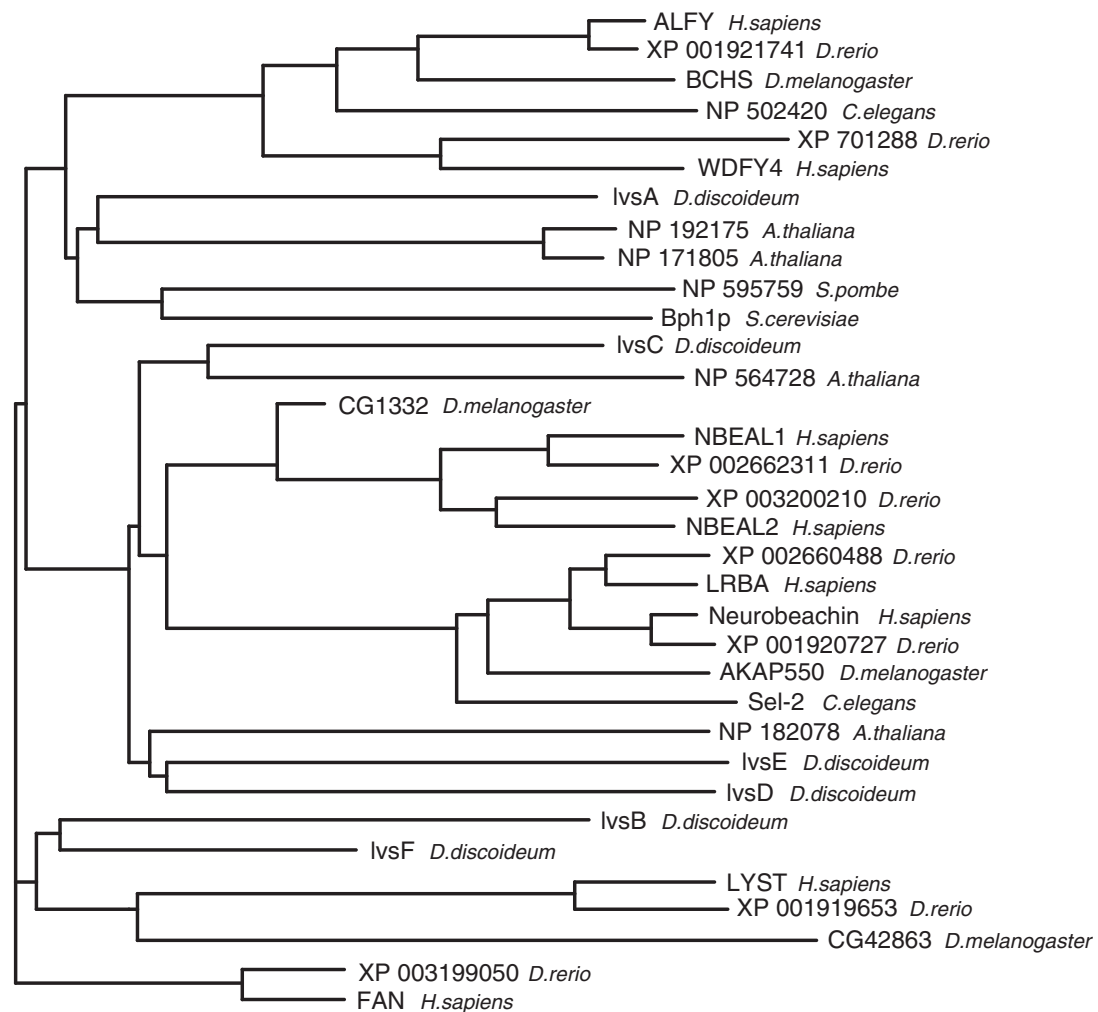

Figure 3 Phylogeny of PH-BEACH-WD40-domain-containing proteins in various species. A search for PH-BEACH-WD40-domain-containing proteins in $H$. sapiens, Arabidopsis thaliana, C. elegans, D. rerio, D. discoideum, D. melanogaster, S. cerevisiae and Schizosaccharomyces pombe resulted in 34 confirmed proteins, which were aligned and assembled phylogenetically 
an Atg5-binding WD40 domain and a FYVE domain binding to PI3P (Figure 2). Depletion of ALFY prevented efficient recruitment of Atg5, as well as LC3, to protein inclusions formed by expression of huntingtin ( $\mathrm{Htt})$-polyQ, suggesting that ALFY might aid the stability of the interaction between LC3 and p62 or itself interact with LC3. ${ }^{48}$ As the Atg5-Atg12 conjugate has E3-ligase activity for conjugation of LC3 to PE, ${ }^{9}$ ALFY may bring Atg5 to the inclusion to facilitate LC3 lipidation, which allows formation of the autophagosomal membrane. This scenario fits with the general concept of selective autophagy, which implies that each cargo is specifically recognized followed by formation of the autophagic membrane closely to the target, thus excluding bulk cytoplasm.

\section{ALFY and p62 in Cytoplasmic Protein Aggregate Clearance}

Protein aggregation starts with misfolded proteins forming oligomeric intermediates that can mature into small protein aggregates and in some cases continue to multimerize into larger aggregates or inclusions. ${ }^{57,58}$ The ubiquitinproteasome system (UPS) is a well-characterized pathway for degradation of misfolded proteins. However, as proteins must pass through the narrow barrel-shaped proteasome, oligomeric complexes and larger protein aggregates are poor substrates for the UPS ${ }^{59}$ and can instead become degraded by autophagy (aggrephagy). The autophagy receptor p62 is a component of protein aggregates associated with several neurodegenerative diseases, supporting the idea of aggregates being toxic species that need to undergo proteolysis. $^{60,61}$ Interestingly, whereas inclusions found in autophagy-deficient cells are positive for both ubiquitin and p62, loss of p62 combined with a deficiency in autophagy greatly reduces the formation of ubiquitin inclusions in mice and flies, ${ }^{62,63}$ indicating that p62 is involved in the formation of protein aggregates. In line with the in vivo data, p62 has been shown to be required for the formation of ubiquitinated protein aggregates, also called p62 bodies, sequestosomes or aggresome-like inducible structures formed upon cellular stress. ${ }^{42,64}$ Importantly, p62 is able to homopolymerize via its N-terminal Phox and Bem1p (PB1) domain and this property, together with its ubiquitin-binding UBA domain, is essential for assembly of aggregation-prone proteins into aggregates that can be degraded by autophagy (Figure 4). ${ }^{14,65}$

Under normal conditions ALFY is predominantly localized to the nucleus and undergoes continuous nucleocytoplasmic shuttling. ${ }^{47}$ Upon cellular stress leading to protein aggregate formation ALFY relocates from the nucleus to cytoplasmic p62 bodies (Figure 4). It is not known what signals ALFY nuclear export upon protein aggregate formation, but p62 might be involved as discussed below. Similar to p62 (and NBR1), depletion of ALFY resulted in a reduced number and size of cytoplasmic p62 bodies and a diffuse localization of ubiquitinated proteins in cells treated with puromycin or the lysosomal proton pump inhibitor Bafilomycin A1. ${ }^{49}$ However, whereas insoluble ubiquitinated proteins almost disappeared in p62 depleted cells, such proteins were still detected in ALFY depleted cells, although at a much lower level than seen in control cells. Moreover, the Drosophila p62 homolog Ref2p was shown to accumulate in ubiquitin-positive inclusions in the brains of flies carrying mutations in the ALFY homolog blue cheese (bchs), demonstrating that ALFY is required for

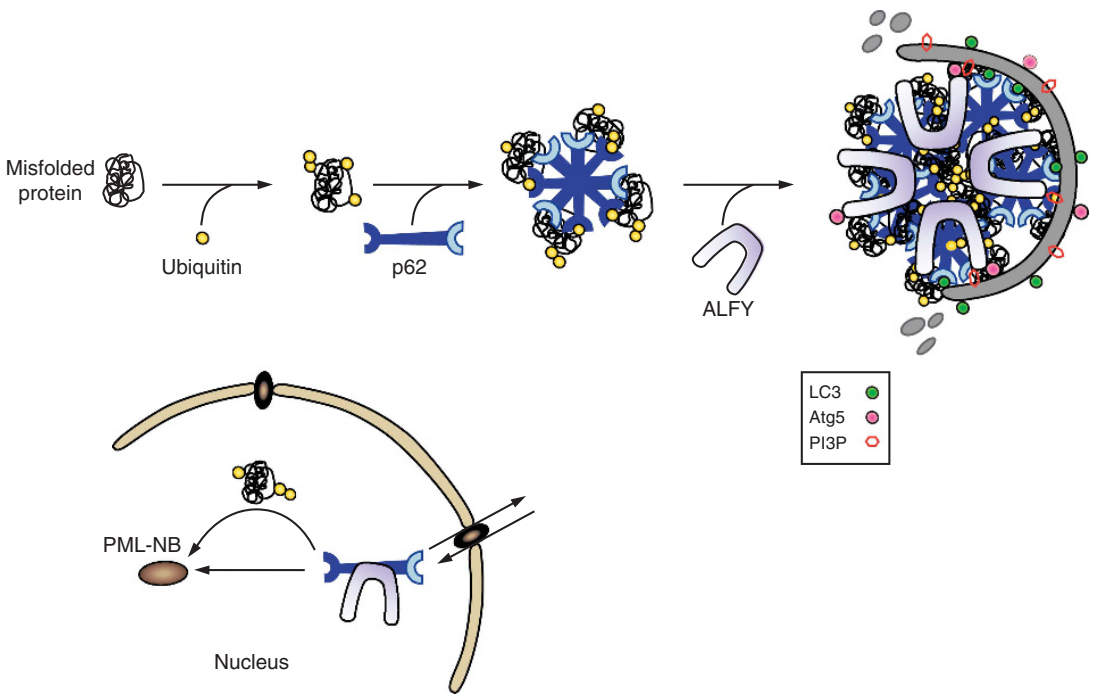

Figure 4 (a) Model for selective autophagy of protein aggregates/p62 bodies (aggrephagy). Aggregate-prone proteins (cargo) become ubiquitinated (ligand) and can be recognized by the ubiquitin-binding domain of the autophagy receptor p62. p62 Further drives the formation of aggregates through its ability to self polymerize via its PB1 domain. ALFY is then recruited through its interaction with p62 and facilitates sequestration of the oligomeric proteins into larger aggregates that can become degraded by autophagy. ALFY is required for recruitment of Atg5-Atg12 to the protein aggregate and through its binding to PI3P in the autophagic membrane it may facilitate binding of Atg5-Atg12 to the membrane associated Atg16L1, creating a complex shown to function as an E3-like ligase to allow conjugation of LC3 to PE in the membrane. ALFY is recruited from the nucleus to cytoplasmic p62 and ubiquitin-positive structures formed upon cellular stress in a p62-dependent manner. In the nucleus, p62 and ALFY are important for recruiting ubiquitinated misfolded proteins into PML-NBs 
the degradation of p62-associated ubiquitinated proteins in vivo. In contrast, bchs did not accumulate in flies lacking a functional Ref2p. Taken together, these data indicate that p62 binds aggregation-prone ubiquitinated proteins and through its PB1 domain facilitates the formation of microaggregates that can be combined and deposited into larger aggregates by ALFY (Figure 4). The large size of ALFY suggests that it works as a scaffolding protein promoting the assembly of p62 bodies and their subsequent degradation by autophagy. Supporting this model is the fact that degradation of ALFY by autophagy depends on p62, whereas p62 is turned over by autophagy independent of ubiquitinated proteins and ALFY. ${ }^{49}$ This might reflect the need for a tight control of the level of p62 as discussed below.

\section{ALFY and p62 in the Nucleus}

Both ALFY and p62 are reported to undergo nucleocytoplasmic shuttling and to localize to promyelocytic leukemia nuclear bodies (PML-NBs) (Figure 4). ${ }^{48,66}$ PML-NBs has been associated with a broad specter of nuclear processes such as transcription, DNA repair, stress, proteolysis and apoptosis. ${ }^{67-69}$ Both p62 and ALFY were found to be required for recruitment of ubiquitinated proteins to these nuclear bodies, ${ }^{49}$ although it cannot be excluded that they rather facilitate recruitment of E3 ligases to PML-NBs for ubiquitination of misfolded proteins. p62 has been suggested to facilitate proteasome recruitment to these sites and thereby help the degradation of misfolded nuclear proteins. ${ }^{66}$ The localization of ALFY to PML-NBs is dependent on p62 and vice versa. ${ }^{49}$ If and how nuclear aggregates are degraded by autophagy is currently not known, but three different models can be proposed; (i) p62 and ALFY bind and export misfolded ubiquitinated proteins through the nuclear pores, (ii) p62 and ALFY associate to nuclear aggregates that become degraded by autophagy during mitosis when the nuclear membrane breaks down and (iii) p62 and ALFY facilitate sorting of nuclear aggregates into nuclear membrane blebs, which become pinched off and degraded by autophagy in a process similar to yeast piecemeal microautophagy of the nucleus. ${ }^{70}$ The finding that the nuclear oncoprotein PML/RARA, expressed in acute promyelocytic leukemia (APL) patients from a chromosomal $t(15,17)$ translocation, is degraded by autophagy in a p62-dependent manner strongly indicate that nuclear aggregate-prone proteins can be targeted by selective autophagy. ${ }^{71}$ It will be exciting to learn if ALFY is also involved in PML/RARA degradation and if/how p62 and/or ALFY facilitate recruitment of PML/RARA from the nucleus to the cytoplasm for degradation by autophagy. Successful treatment of APL patients with the drugs all-trans retinoic acid (ATRA) or arsenic trioxide (ATO) relies on their ability to induce proteolytic degradation of PML/RARA, and we recently reported this to be autophagy dependent. ${ }^{72}$ Thus, manipulation of autophagy or p62/ALFY levels may improve therapy for APL patients that have become resistant to ATRA/ATO.

\section{ALFY and Neurodegeneration}

Drosophila lacking the ALFY homolog bchs have a neurodegenerative phenotype characterized by accumulation of insoluble ubiquitin-positive inclusions in their brain and a reduced life span, ${ }^{73}$ indicating that $A L F Y$ is important for degradation of aggregation-prone ubiquitinated proteins also in vivo. Confocal immunofluorescence and biochemical analysis revealed that ALFY forms a complex with polyQ protein-containing inclusions associated with Huntington's disease (HD). Furthermore, ALFY was required for efficient clearance of such inclusions by autophagy in two different tetregulatable cell lines (HeLa and Neuro2a). ${ }^{48}$ In contrast, ALFY depletion had no effect on the clearance of soluble HttQ25 proteins, indicating that ALFY is specifically required for turnover of aggregation-prone Htt proteins. Moreover, double knock down of ALFY and Atg5 did not result in further inhibition of clearance, suggesting that they function in a common autophagy pathway. Most intriguingly, whereas loss of ALFY inhibits inclusion clearance, overexpression of an ALFY C-terminal fragment (residues 2981-3526) caused a significant reduction in the number of $\mathrm{Htt}$ polyQ inclusions in rat cortical primary neurons, as well as in HeLa and Neuro2a cells. This rescue effect was not detected when the Atg5binding site in ALFY, the WD40 domain, was mutated. Furthermore, overexpression of either full length or the C-terminal part of bchs led to neuroprotection in a Drosophila eye model of polyglutamine toxicity. ${ }^{48}$ These results indicate that increased ALFY levels can drive elimination of the aggregated proteins, which help to re-establish cell homeostasis. From inducible mouse models of neurodegeneration it is known that a continuous expression of the pathological protein is required to maintain inclusions and the neurodegenerative phenotype, raising the possibility that diseases caused by aggregating proteins (such as HD) may be reversible. ${ }^{74-82}$

It seems like the role of ALFY in aggregate clearance can be extended to other disease-associated aggregate-prone proteins. ALFY depletion inhibited clearance of mutant $\alpha$-synuclein A53T, linked to Parkinson's disease, and co-immunoprecipitation experiments revealed that $\alpha$-synuclein is detected in the same complex as Atg5 and ALFY. ${ }^{48}$ Furthermore, ALFY, as p62, ${ }^{34}$ seems to be involved in selective autophagy of other ubiquitinated structures, such as the midbody ring structure (Isakson et al., unpublished).

\section{ALFY and Regulation of Selective Autophagy}

The mechanisms involved in regulation of selective autophagy are largely unknown, but is likely controlled at the level of autophagy receptors and adaptors. The core Atg machinery is required for both non-selective and selective autophagy and a competition between these two types of autophagy for the same molecular machinery could be detrimental for cells undergoing starvation. Tissue expression levels of ALFY (highest in the brain and lowest in the liver) ${ }^{47}$ inversely correlate with responsiveness to starvation and suggest that ALFY may be especially important in neurons, non-dividing cells in which removal of toxic aggregation-prone proteins is essential for survival. Thus, regulation of the ALFY level or localization might be a way for the cell to regulate starvationinduced autophagy versus aggrephagy. ${ }^{83}$ ALFY is an ideal candidate for such a regulator, as it mainly localizes to the nucleus under normal conditions and becomes recruited to 
cytoplasmic protein aggregates upon cellular stress. ${ }^{47}$ Thus, keeping ALFY in the nucleus when the need for selective autophagy is low would be a way of avoiding toxicity under conditions where non-selective autophagy may be necessary. Interestingly, several other autophagy-related proteins also undergo nucleocytoplasmic shuttling, but in general little is known about the functional consequence of their nuclear shuttling. However, nuclear export of Beclin1 seems to be required for its autophagy and tumor suppressor function. ${ }^{84}$

Nucleocytoplasmic shuttling is normally mediated by importin- $\alpha$ binding to a nuclear localization signal (NLS) and exportin-1/CRM1 binding to a nuclear export signal (NES) of the shuttling protein. ALFY contains both NLS and NES signals (Pankiv et al., unpublished). Interestingly, p62 was found to facilitate nuclear export of ALFY, ${ }^{49}$ suggesting that p62 might signal when aggrephagy needs to be activated. p62 is mainly localized in the cytoplasm and its nuclear import is modulated by phosphorylation at or near one of its NLSs. ${ }^{66}$ It will be interesting to elucidate whether nucleocytoplasmic shuttling of ALFY is also regulated by post-translational modifications.

\section{ALFY Homologs}

As described above, ALFY is a member of the $\mathrm{PH}-\mathrm{BEACH}$ WD40 protein family. Just as the $\mathrm{BEACH}$ domain is very well conserved through the human protein family, it is also conserved in other species (Figure 3). Families of $\mathrm{BEACH}$ proteins have emerged by duplication events throughout eukayotic evolution correlating with increases in organismal complexity, from one BEACH protein in yeasts to two in Caenorhabditis elegans, four in Drosophila melanogaster and eight in Homo sapiens and Dario. rerio. The zebrafish D. rerio looks like an attractive model organism to study human $\mathrm{BEACH}$ proteins as it contains closely related homologs to each human protein.

Putative ALFY orthologues can be found in Drosophila (blue cheese, BCHS), C. elegans (NP_502420) and in zebrafish (XP_001921741). Whereas nothing is known about the $C$. elegans and zabrafish ortholouges, bchs has been quite well characterized. bchs has extensive homology to ALFY throughout the entire sequence and consistent with the mouse tissue expression profile of ALFY (highest in the brain), ${ }^{47}$ bchs seems to be selectively expressed in the fly brain. ${ }^{85}$ Moreover, adult flies lacking bchs accumulate ubiquitin-positive inclusions and display a neurodegenerative phenotype, ${ }^{73}$ in line with the proposed function of ALFY in autophagic degradation of aggregate-prone proteins. In a screen for genetic modifiers of a gain-of-function (GOF) rough eye phenotype caused by overexpression of bchs, the small GTPase Rab11 was found to enhance this phenotype. Moreover, loss of bchs was found to strongly suppress developmental defects exhibited by rab11 loss-of-function mutants, ${ }^{85}$ suggesting that bchs functions during Drosophila development as an antagonist of Rab11-mediated vesicle trafficking. Interestingly, the presence of a putative TBC domain in ALFY/bchs might indicate that bchs negatively regulates Rab11 by promoting its GTPase activity. A similar GOF screen identified genetic interactions with proteins involved in autophagy and endocytic trafficking, as well in the SUMO and ubiquitin signaling pathways. ${ }^{86}$

Of the other members of the ALFY subfamily, deletion of the only yeast $B E A C H$ gene, BPH1 in Saccharomyces cerevisiae resulted in a vacuolar protein sorting phenotype characterized by increased carboxypeptidase $Y$ secretion and missorting of alkaline phosphatase, indicating that Bph1p is important for sorting from the Golgi to the vacuole. ${ }^{87}$ From our phylogenetic analysis Bph1p seems to be most closely related to the ALFY subfamily of BEACH proteins (Figure 3 ), but its role in selective autophagy has not been analyzed. In Dictyostelium discoideum, IVSA is the only BEACH protein of the ALFY subfamily and this protein has been implicated in contractile vacuole function and cytokinesis. ${ }^{88}$ The human protein most closely related to ALFY is the WD repeat and FYVE domaincontaining protein 4 (WDFY4) which despite of its name does not contain a complete FYVE domain. It is expressed in immune tissue and mutations in the coding genes have been linked to systemic lupus erythematosus. ${ }^{89}$ The fact that there seems to be only one variant of ALFY/WDFY4 in C. elegans and $D$. melanogaster suggests that ALFY and WDFY4 have arisen by a gene duplication and therefore might have similar functions. Although little is known about most $\mathrm{PH}-\mathrm{BEACH}-$ WD40-domain-containing proteins, the ones that have been studied so far are all involved in protein sorting related to establishing, maintaining and/or transporting vesicular systems, so it seems likely that this is a defining characteristic of the protein family.

\section{Concluding Remarks}

Autophagy was long considered a non-selective bulk protein degradation system, but recent work has demonstrated that it is also a highly selective process involving recognition of specific cargo by autophagy receptors, which together with adaptor proteins link the cargo to the core autophagy machinery to allow its sequestration by autophagic membranes. Although this is an active area of study, there are still several important questions that remains to be elucidated. For example, how are the various types of selective autophagy regulated? What are the ligands involved in targeting different cargo for selective autophagy? Which autophagy receptors and adaptor proteins are involved in recognition of the various types of cargo? Increased knowledge about the various types of selective autophagy is important as they serve a protective function by removing intracellular pathogens, damaged proteins and organelles that could be potentially dangerous for the cell.

Accumulation of ubiquitinated protein aggregates is a common feature of many neurodegenerative disorders, as well as a number of other protein aggregation diseases (proteinopathies) including those affecting the muscles, heart and liver. Induced autophagy enhance the degradation of aggregate-prone proteins and alleviate their cytotoxic effects across many of these diseases. ${ }^{90}$ It is therefore tempting to speculate that manipulation of aggrephagy through regulation of autophagy receptors and/or adaptors could improve therapy against many proteinopathies. The p62-interacting adaptor protein ALFY is required for aggrephagy, but not for autophagic degradation of bulk cytosol in response to 
starvation. As the tissue expression levels of ALFY inversely correlate with responsiveness to starvation, regulation of ALFY might be a way for the cell to regulate starvationinduced versus selective autophagy. Indeed, overexpression of ALFY or its C-terminal p62-, Atg5- and PI3P-binding region was found to decrease the number of protein inclusions in primary neurons expressing polyQ $\mathrm{Htt}$ and be neuroprotective in a Drosophila eye model of polyQ disease, indicating that increased ALFY levels stimulate aggrephagy. ${ }^{48}$

ALFY is mostly localized in the nucleus under normal conditions and becomes recruited to the cytoplasm during conditions favoring the formation of protein aggregates. ${ }^{47}$ Keeping ALFY in the nucleus under normal conditions might be important, as cytoplasmic ALFY, through its interactions with the core autophagic machinery might prevent the cell from mounting an efficient autophagic response upon starvation. How this is regulated is not known, but p62 might signal the need for elevated levels of aggrephagy. Thus, regulation of ALFY and/or p62 nucleocytoplasmic shuttling could be exploited therapeutically to enhance clearance of protein aggregates. It will be interesting to learn whether ALFY, as yeast Atg11, is also involved in other forms of selective autophagy and whether other adaptor proteins of mammalian autophagy exist.

\section{Conflict of Interest}

The authors declare no conflict of interest.

Acknowledgements. $\mathrm{Pl}$ is supported by grants from the Research Council of Norway (RCN). AS is supported by the RCN, by the Norwegian Cancer Society and by the South-Eastern Norway Regional Health Authority. We thank Drs Terje Johansen and Ai Yamamoto for critical reading of the manuscript and Carina VS Knudsen for help with the figures.

1. Yang Z, Klionsky DJ. Mammalian autophagy: core molecular machinery and signaling regulation. Curr Opin Cell Biol 2010; 22: 124-131.

2. Mizushima N. The role of the Atg1/ULK1 complex in autophagy regulation. Curr Opin Cell Biol 2010; 22: 132-139.

3. Simonsen A, Tooze SA. Coordination of membrane events during autophagy by multiple class III PI3-kinase complexes. J Cell Biol 2009; 186: 773-782.

4. Webber JL, Tooze SA. New insights into the function of Atg9. FEBS Lett 2010; 584 1319-1326.

5. Ichimura $\mathrm{Y}$, Kirisako T, Takao T, Satomi $\mathrm{Y}$, Shimonishi $\mathrm{Y}$, Ishihara $\mathrm{N}$ et al. A ubiquitin-like system mediates protein lipidation. Nature 2000; 408: 488-492.

6. Mizushima N, Noda T, Yoshimori T, Tanaka Y, Ishii T, George MD et al. A protein conjugation system essential for autophagy. Nature 1998; 395: 395-398.

7. Geng J, Klionsky DJ. The Atg8 and Atg12 ubiquitin-like conjugation systems in macroautophagy. 'Protein modifications: beyond the usual suspects' review series. EMBO Rep 2008; 9: 859-864.

8. Fujita N, Itoh T, Omori H, Fukuda M, Noda T, Yoshimori T. The Atg16 L complex specifies the site of LC3 lipidation for membrane biogenesis in autophagy. Mol Biol Cell 2008; 19 : 2092-2100.

9. Hanada T, Noda NN, Satomi Y, Ichimura Y, Fujioka Y, Takao T et al. The Atg12-Atg5 conjugate has a novel E3-like activity for protein lipidation in autophagy. J Biol Chem 2007; 282: 37298-37302.

10. Lynch-Day MA, Klionsky DJ. The Cvt pathway as a model for selective autophagy. FEBS Lett 2010; 584: 1359-1366.

11. Klionsky DJ, Cuervo AM, Dunn WA Jr, Levine B, van der Klei I, Seglen PO. How shall I eat thee? Autophagy 2007; 3: 413-416.

12. Noda NN, Kumeta $H$, Nakatogawa $H$, Satoo $K$, Adachi $W$, Ishii $J$ et al. Structural basis of target recognition by Atg8/LC3 during selective autophagy. Genes Cells 2008; 13 1211-1218

13. Pankiv S, Clausen TH, Lamark T, Brech A, Bruun JA, Outzen H et al. p62/SQSTM1 binds directly to Atg8/LC3 to facilitate degradation of ubiquitinated protein aggregates by autophagy. J Biol Chem 2007; 282: 24131-24145.

14. Ichimura Y, Kumanomidou T, Sou YS, Mizushima T, Ezaki J, Ueno T et al. Structural basis for sorting mechanism of p62 in selective autophagy. J Biol Chem 2008; 283 22847-22857.
15. Shpilka T, Weidberg H, Pietrokovski S, Elazar Z. Atg8: an autophagy-related ubiquitin-like protein family. Genome Biol 2011; 12: 226.

16. Satoo K, Noda NN, Kumeta H, Fujioka Y, Mizushima N, Ohsumi $Y$ et al. The structure of Atg4B-LC3 complex reveals the mechanism of LC3 processing and delipidation during autophagy. EMBO J 2009; 28: 1341-1350.

17. Yamaguchi $M$, Noda NN, Nakatogawa $H$, Kumeta $H$, Ohsumi $Y$, Inagaki $F$. Autophagy-related protein 8 (Atg8) family interacting motif in Atg3 mediates the Atg3-Atg8 interaction and is crucial for the cytoplasm-to-vacuole targeting pathway. J Biol Chem 2010; 285: 29599-29607.

18. Pankiv S, Alemu EA, Brech A, Bruun JA, Lamark T, Overvatn A et al. FYCO1 is a Rab7 effector that binds to LC3 and PI3P to mediate microtubule plus end-directed vesicle transport. J Cell Biol 2010; 188: 253-269.

19. Itoh T, Kanno E, Uemura T, Waguri S, Fukuda M. OATL1, a novel autophagosomeresident Rab33B-GAP, regulates autophagosomal maturation. J Cell Biol 2011; 192: 839-853.

20. Gao C, Cao W, Bao L, Zuo W, Xie G, Cai T et al. Autophagy negatively regulates Wnt signalling by promoting Dishevelled degradation. Nat Cell Biol 2010; 12: 781-790.

21. Dupont N, Lacas-Gervais S, Bertout J, Paz I, Freche B, Van Nhieu GT et al. Shigella phagocytic vacuolar membrane remnants participate in the cellular response to pathogen invasion and are regulated by autophagy. Cell Host Microbe 2009; 6: 137-149.

22. Yoshikawa $Y$, Ogawa M, Hain T, Yoshida M, Fukumatsu M, Kim M et al. Listeria monocytogenes ActA-mediated escape from autophagic recognition. Nat Cell Biol 2009; 11: $1233-1240$.

23. Zheng YT, Shahnazari S, Brech A, Lamark T, Johansen T, Brumell JH. The adaptor protein p62/SQSTM1 targets invading bacteria to the autophagy pathway. J Immunol 2009; 183: 5909-5916.

24. Thurston TL, Ryzhakov G, Bloor S, von Muhlinen N, Randow F. The TBK1 adaptor and autophagy receptor NDP52 restricts the proliferation of ubiquitin-coated bacteria. Nat Immunol 2009; 10: 1215-1221.

25. Wild P, Farhan H, McEwan DG, Wagner S, Rogov VV, Brady NR et al. Phosphorylation of the autophagy receptor optineurin restricts Salmonella growth. Science 2011; 333: 228-233.

26. Orvedahl A, Jr RS, Xiao G, Ng A, Zou Z, Tang Y et al. Image-based genome-wide siRNA screen identifies selective autophagy factors. Nature 2011; 480: 113-117.

27. Okamoto K, Kondo-Okamoto N, Ohsumi Y. Mitochondria-anchored receptor Atg32 mediates degradation of mitochondria via selective autophagy. DevCell 2009; 17: 87-97.

28. Kanki T, Wang K, Cao Y, Baba M, Klionsky DJ. Atg32 is a mitochondrial protein that confers selectivity during mitophagy. DevCell 2009; 17: 98-109.

29. Novak I, Kirkin V, McEwan DG, Zhang J, Wild P, Rozenknop A et al. Nix is a selective autophagy receptor for mitochondrial clearance. EMBO Rep 2010; 11: 45-51.

30. Geisler S, Holmstrom KM, Skujat D, Fiesel FC, Rothfuss OC, Kahle PJ et al. PINK1/Parkinmediated mitophagy is dependent on VDAC1 and p62/SQSTM1. Nat Cell Biol 2010; 12: 119-131.

31. Ding WX, Ni HM, Li M, Liao Y, Chen X, Stolz DB et al. Nix is critical to two distinct phases of mitophagy, reactive oxygen species-mediated autophagy induction and Parkinubiquitin-p62-mediated mitochondrial priming. J Biol Chem 2010; 285: 27879-27890.

32. Farre JC, Manjithaya R, Mathewson RD, Subramani S. PpAtg30 tags peroxisomes for turnover by selective autophagy. Dev Cell 2008; 14: 365-376.

33. Kim PK, Hailey DW, Mullen RT, Lippincott-Schwartz J. Ubiquitin signals autophagic degradation of cytosolic proteins and peroxisomes. Proc Natl Acad Sci USA 2008; 105 : 20567-20574

34. Pohl C, Jentsch S. Midbody ring disposal by autophagy is a post-abscission event of cytokinesis. NatCell Biol 2009; 11: 65-70.

35. Kuo TC, Chen CT, Baron D, Onder TT, Loewer S, Almeida S et al. Midbody accumulation through evasion of autophagy contributes to cellular reprogramming and tumorigenicity. Nat Cell Biol 2011; 13: 1214-1223.

36. Jiang S, Wells CD, Roach PJ. Starch-binding domain-containing protein 1 (Stbd1) and glycogen metabolism: Identification of the Atg8 family interacting motif (AIM) in Stbd1 required for interaction with GABARAPL1. Biochem Biophys Res Commun 2011; 413 : 420-425.

37. Watanabe Y, Noda NN, Kumeta H, Suzuki K, Onsumi Y, Inagaki F. Selective transport of alpha-mannosidase by autophagic pathways: structural basis for cargo recognition by Atg19 and Atg34. J Biol Chem 2010; 285: 30026-30033.

38. Suzuki K, Kondo C, Morimoto M, Ohsumi Y. Selective transport of alpha-mannosidase by autophagic pathways: identification of a novel receptor, Atg34p. J Biol Chem 2010; 285: 30019-30025.

39. Manjithaya R, Nazarko TY, Farre JC, Subramani S. Molecular mechanism and physiological role of pexophagy. FEBS Lett 2010; 584: 1367-1373.

40. Johansen T, Lamark T. Selective autophagy mediated by autophagic adapter proteins. Autophagy 2011; 7: 279-296.

41. Randow F. How cells deploy ubiquitin and autophagy to defend their cytosol from bacterial invasion. Autophagy 2011; 7: 304-309.

42. Bjorkoy G, Lamark T, Brech A, Outzen H, Perander M, Overvatn A et al. p62/SQSTM1 forms protein aggregates degraded by autophagy and has a protective effect on huntingtin-induced cell death. J Cell Biol 2005; 171: 603-614.

43. Monastyrska I, Shintani T, Klionsky DJ, Reggiori F. Atg11 directs autophagosome cargoes to the PAS along actin cables. Autophagy 2006; 2: 119-121. 
44. Okamoto K, Kondo-Okamoto N, Ohsumi Y. Mitochondria-anchored receptor Atg32 mediates degradation of mitochondria via selective autophagy. Dev Cell 2009; 17: 87-97.

45. Nazarko TY, Farre JC, Subramani S. Peroxisome size provides insights into the function of autophagy-related proteins. Mol Biol Cell 2009; 20: 3828-3839.

46. He C, Song H, Yorimitsu T, Monastyrska I, Yen W-L, Legakis JE et al. Recruitment of Atg9 to the preautophagosomal structure by Atg11 is essential for selective autophagy in budding yeast. J Cell Biol 2006; 175: 925-935.

47. Simonsen A, Birkeland HC, Gillooly DJ, Mizushima N, Kuma A, Yoshimori T et al. Alfy, a novel FYVE-domain-containing protein associated with protein granules and autophagic membranes. J Cell Sci 2004; 117(Pt 18): 4239-4251.

48. Filimonenko M, Isakson $\mathrm{P}$, Finley KD, Anderson M, Jeong $\mathrm{H}$, Melia TJ et al. The selective macroautophagic degradation of aggregated proteins requires the PI3P-binding protein Alfy. Mol Cell 2010; 38: 265-279.

49. Clausen TH, Lamark T, Isakson P, Finley K, Larsen KB, Brech A et al. p62/SQSTM1 and ALFY interact to facilitate the formation of $p 62$ bodies/ALIS and their degradation by autophagy. Autophagy 2010; 6: 330-344.

50. Fukuda M. TBC. Proteins: GAPs for mammalian small GTPase Rab? Bioscience Rep 2011; 31: 159-168.

51. Stenmark H, Aasland R, Driscoll PC. The phosphatidylinositol 3-phosphate-binding FYVE finger. FEBS Lett 2002; 513: 77-84.

52. Gillooly DJ, Morrow IC, Lindsay M, Gould R, Bryant NJ, Gaullier JM et al. Localization of phosphatidylinositol 3-phosphate in yeast and mammalian cells (in process citation). EMBO J 2000; 19: 4577-4588.

53. Xu C, Min J. Structure and function of WD40 domain proteins. Protein \& cell 2011; 2 202-214.

54. Jogl G, Shen Y, Gebauer D, Li J, Wiegmann K, Kashkar H et al. Crystal structure of the $\mathrm{BEACH}$ domain reveals an unusual fold and extensive association with a novel $\mathrm{PH}$ domain EMBO J 2002; 21: 4785-4795.

55. Haubert D, Gharib N, Rivero F, Wiegmann K, Hosel M, Kronke M et al. Ptdlns $(4,5)$ P-restricted plasma membrane localization of FAN is involved in TNF-induced actin reorganization. EMBO J 2007; 26: 3308-3321.

56. Adam-Klages S, Adam D, Wiegmann K, Struve S, Kolanus W, Schneider-Mergener J et al. FAN a novel WD-repeat protein, couples the p55 TNF-receptor to neutral sphingomyelinase. Cell 1996; 86: 937-947.

57. Kopito RR. Aggresomes, inclusion bodies and protein aggregation. Trends Cell Biol 2000; 10: $524-530$.

58. Grune T, Jung T, Merker K, Davies KJ. Decreased proteolysis caused by protein aggregates, inclusion bodies, plaques, lipofuscin, ceroid, and 'aggresomes' during oxidative stress, aging, and disease. Int J Biochem Cell Biol 2004; 36: 2519-2530.

59. Verhoef LG, Lindsten K, Masucci MG, Dantuma NP. Aggregate formation inhibits proteasomal degradation of polyglutamine proteins. Hum Mol Genet 2002; 11: 2689-2700.

60. Gal J, Strom AL, Kwinter DM, Kilty R, Zhang J, Shi P et al. Sequestosome 1/p62 links familial ALS mutant SOD1 to LC3 via an ubiquitin-independent mechanism. J Neurochem 2009; 111: 1062-1073

61. Holm IE, Englund E, Mackenzie IR, Johannsen $P$, Isaacs AM. A reassessment of the neuropathology of frontotemporal dementia linked to chromosome 3. J Neuropathol Exp Neurol 2007; 66: 884-891.

62. Komatsu M, Waguri S, Koike M, Sou YS, Ueno T, Hara T et al. Homeostatic levels of p62 control cytoplasmic inclusion body formation in autophagy-deficient mice. Cell 2007; 131 $1149-1163$.

63. Nezis IP, Simonsen A, Sagona AP, Finley K, Gaumer S, Contamine D et al. Ref(2)P, the Drosophila melanogaster homologue of mammalian $\mathrm{p} 62$, is required for the formation of protein aggregates in adult brain. J Cell Biol 2008; 180: 1065-1071.

64. Szeto J, Kaniuk NA, Canadien V, Nisman R, Mizushima N, Yoshimori T et al. ALIS are stress-induced protein storage compartments for substrates of the proteasome and autophagy. Autophagy 2006; 2: 189-199.

65. Lamark T, Perander M, Outzen H, Kristiansen K, Overvatn A, Michaelsen E et al. Interaction codes within the family of mammalian Phox and Bem1p domain-containing proteins. J Biol Chem 2003; 278: 34568-34581.

66. Pankiv S, Lamark T, Bruun JA, Overvatn A, Bjorkoy G, Johansen T. Nucleocytoplasmic shuttling of p62/SQSTM1 and its role in recruitment of nuclear polyubiquitinated proteins to promyelocytic leukemia bodies. J Biol Chem 2010; 285: 5941-5953.

67. Bernardi R, Pandolfi PP. Structure, dynamics and functions of promyelocytic leukaemia nuclear bodies. Nat Rev Mol Cell Biol 2007; 8: 1006-1016.
68. Dellaire G, Bazett-Jones DP. PML nuclear bodies: dynamic sensors of DNA damage and cellular stress. BioEssays 2004; 26: 963-977.

69. Zhong S, Salomoni P, Pandolfi PP. The transcriptional role of PML and the nuclear body. Nat Cell Biol 2000; 2: E85-E90.

70. Krick R, Muehe Y, Prick T, Bremer S, Schlotterhose P, Eskelinen EL et al. Piecemeal microautophagy of the nucleus requires the core macroautophagy genes. Mol Biol Cell 2008; 19: 4492-4505.

71. Wang Z, Cao L, Kang R, Yang M, Liu L, Zhao Y et al. Autophagy regulates myeloid cell differentiation by p62/SQSTM1-mediated degradation of PML-RARalpha oncoprotein. Autophagy 2011; 7: 401-411

72. Isakson $\mathrm{P}$, Bjoras $\mathrm{M}$, Boe SO, Simonsen A. Autophagy contributes to therapyinduced degradation of the PML/RARA oncoprotein. Blood 2010; 116(13): 2324-2331.

73. Finley KD, Edeen PT, Cumming RC, Mardahl-Dumesnil MD, Taylor BJ, Rodriguez $\mathrm{MH}$ et al. blue cheese mutations define a novel, conserved gene involved in progressive neural degeneration. J Neurosci 2003; 23: 1254-1264.

74. DiFiglia M, Sena-Esteves M, Chase K, Sapp E, Pfister E, Sass M et al. Therapeutic silencing of mutant huntingtin with siRNA attenuates striatal and cortical neuropathology and behavioral deficits. Proc Natl Acad Sci USA 2007; 104: 17204-17209.

75. Lin X, Parisiadou L, Gu XL, Wang L, Shim H, Sun L et al. Leucine-rich repeat kinase 2 regulates the progression of neuropathology induced by Parkinson's-disease-related mutant alpha-synuclein. Neuron 2009; 64: 807-827.

76. Mallucci G, Dickinson A, Linehan J, Klohn PC, Brandner S, Collinge J. Depleting neuronal PrP in prion infection prevents disease and reverses spongiosis. Science 2003 302: 871-874.

77. Regulier E, Trottier Y, Perrin V, Aebischer P, Deglon N. Early and reversible neuropathology induced by tetracycline-regulated lentiviral overexpression of mutan huntingtin in rat striatum. Hum Mol Genet 2003; 12: 2827-2836.

78. Southwell AL, Ko J, Patterson PH. Intrabody gene therapy ameliorates motor, cognitive, and neuropathological symptoms in multiple mouse models of Huntington's disease. J Neurosci 2009; 29: 13589-13602.

79. Wang CE, Zhou H, McGuire JR, Cerullo V, Lee B, Li SH et al. Suppression of neuropil aggregates and neurological symptoms by an intracellular antibody implicates the cytoplasmic toxicity of mutant huntingtin. J Cell Biol 2008; 181: 803-816.

80. Xia $\mathrm{H}$, Mao $\mathrm{Q}$, Eliason $\mathrm{SL}$, Harper $\mathrm{SQ}$, Martins $\mathrm{H}$, Orr $\mathrm{HT}$ et al. RNAi suppresses polyglutamine-induced neurodegeneration in a model of spinocerebellar ataxia. Nat Med 2004; 10: 816-820.

81. Yamamoto A, Lucas JJ, Hen R. Reversal of neuropathology and motor dysfunction in a conditional model of Huntington's disease. Cell 2000; 101: 57-66.

82. Zu T, Duvick LA, Kaytor MD, Berlinger MS, Zoghbi HY, Clark HB et al. Recovery from polyglutamine-induced neurodegeneration in conditional SCA1 transgenic mice. J Neurosci 2004; 24: 8853-8861.

83. Yamamoto A, Simonsen A. Alfy-dependent elimination of aggregated proteins by macroautophagy: can there be too much of a good thing? Autophagy $2011 ; 7$ 346-350.

84. Liang XH, Yu J, Brown K, Levine B. Beclin 1 contains a leucine-rich nuclear export signal that is required for its autophagy and tumor suppressor function. Cancer Res 2001; 61: 3443-3449.

85. Khodosh R, Augsburger A, Schwarz TL, Bchs Garrity PA. a BEACH domain protein, antagonizes Rab11 in synapse morphogenesis and other developmental events. Development 2006; 133: 4655-4665.

86. Simonsen A, Cumming RC, Lindmo K, Galaviz V, Cheng S, Rusten TE et al. Genetic modifiers of the Drosophila blue cheese gene link defects in lysosomal transport with decreased life span and altered ubiquitinated-protein profiles. Genetics 2007; 176 1283-1297.

87. Shiflett SL, Vaughn MB, Huynh D, Kaplan J, Ward DM. Bph1p the Saccharomyces cerevisiae homologue of $\mathrm{CHS} 1 /$ beige, functions in cell wall formation and protein sorting. Traffic 2004; 5: 700-710.

88. Wu W-I, Yajnik J, Siano M, De Lozanne A. Structure-Function Analysis of the BEACH Protein LvsA. Traffic 2004; 5: 346-355.

89. Yang W, Shen N, Ye D-Q, Liu Q, Zhang Y, Qian X-X et al. Genome-Wide Association Study in Asian populations identifies variants in ETS1 and WDFY4 associated with systemic lupus erythematosus. PLoS Genet 2010; 6: e1000841.

90. Yamamoto A, Simonsen A. The elimination of accumulated and aggregated proteins: a role for aggrephagy in neurodegeneration. Neurobiol Dis 2011; 43: 17-28. 